\title{
EL AFECTO EN LA RESOLUCIÓN DE PROBLEMAS DE MATEMÁTICA
}

\author{
The Affect on Mathematics Problem Solving
}

Oswaldo Jesús Martínez-Padrón'

ommadail@gmail.com

${ }^{1}$ Universidad Pedagógica Experimental Libertador, Venezuela

Fecha de recepción: 23/5/2020

Fecha de aprobación: 15/07/2020

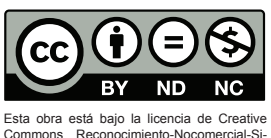

Esta obra está bajo la licencia de Creative
Commons Reconocimiento-Nocomercial-S

nobraDerivada 4.0 Internacional.

ISSN (impreso): 2636-2139

ISSN (en línea): 2636-2147

Sitio web: https://revistas.isfodosu.edu.do/recie

\section{Resumen}

Resolver problemas matemáticos es un asunto que debe trascender las tradicionales ejercitaciones rutinarias y descontextualizadas que forman parte de las clases impartidas en muchas aulas donde se enseña Matemática. Tales rutinas suelen distar de la posibilidad de desarrollar potencialidades cognitivas, metacognitivas, comportamentales, motivacionales y afectivas que vayan a favor de los resolutores. Eso obliga a proveer a los estudiantes de los recursos necesarios para que puedan concebir planes de acción donde se establezcan relaciones posibles entre los datos e incógnitas del problema hasta concretar su posible solución. En este sentido, se presenta esta investigación documental, apoyada en un análisis de contenidos, cuyo objetivo está dirigido a analizar una serie de aspectos afectivos relacionados con la resolución de problemas matemáticos, tomando en cuenta lo que acontece antes, durante o después de intentar resolver tales problemas. Entre los hallazgos relacionados con los asuntos afectivos afines con esta resolución, por parte de los estudiantes, destaca la presencia de incompetencias matemáticas aprendidas, aversión hacia la Matemática y ausencia de resiliencia matemática, lo cual obliga a revisar, adecuar y potenciar, tanto a nivel cognitivo como afectivo, el conocimiento profesional que tienen los docentes que enseñan esta asignatura. Solventar ese problema de formación profesional abre la posibilidad de atender particularidades ligadas con creencias, actitudes, motivaciones, determinaciones y emociones que juegan un papel preponderante al momento de resolver cualquier problema matemático.

Palabras clave: actitudes, afecto, aprendizaje, educación matemática, emociones, resolución de problemas.

\section{Abstract}

Solving mathematical problems is a matter that must go beyond the traditional and decontextualized exercises that are a part of the classes that take place in many classrooms where Mathematics is taught. Such routines are usually different from the possibility of developing cognitive, metacognitive, behavioral, motivational, and affective potentialities that go in favor of the resolvers. Taking this into consideration, providing students with the necessary resources so that they can devise action plans where possible relationships between the data and the unknowns of the problem are established until the solution of the problem becomes a necessity. In this sense, this documentary research is presented, supported by a content analysis, with the objective of analyzing a series of affective aspects related to the resolution of mathematical problems, taking into account what happens before, during and after trying to solve such problems. Among the findings regarding the affective issues related to this resolution, on the part of the students, the presence of learned mathematical incompetence, aversion towards Mathematics and absence of Mathematical Resilience stands out, which forces to review, adapt, and enhance, in both cognitive and affective terms the professional knowledge that teachers who impart this subject have. Solving this problem of professional training opens the possibility of attending to particularities linked to beliefs, attitudes, motivations, determinations, and emotions that play a preponderant role when solving any mathematical problem.

Keywords: attitudes, affection, learning, Mathematical Education, emotions, problem solving. 


\section{MARTÍNEZ-PADRÓN}

El afecto en la resolución de problemas de Matemática

\section{Introducción}

La resolución de cualquier problema de Matemática solicita del resolutor la posesión de ciertas pericias que le permitan encontrar, exitosamente, su solución, en un tiempo oportuno. Resolver ejercicios de Matemática también demanda la puesta en escena de ciertas competencias, pero, a diferencia de los problemas, pueden resolverse con "la reproducción sistemática de algoritmos, métodos y reglas ya empleadas en el ejemplo o en otros ejercicios semejantes o parecidos que son desarrollados a continuación del ejemplo" (Martínez-Padrón \& González, 2007, p. 46).

En vista de que el corazón de la Matemática son los problemas (Halmos, 2018; González; 2020) y su resolución no es un asunto meramente cognitivo (Polya, 1965), sino metacognitivo, social y afectivo (García-González \& Martínez-Padrón, 2020; Martínez-Padrón, 2008, 2016), entonces en ese asunto intervienen variados factores que deben ser considerados no solo por los que intentan resolverlos, sino por quienes enseñan Matemática.

Aunque existen investigaciones al respecto, el problema del aprendizaje de la Matemática continúa vigente, sobre todo cuando se intenta resolver problemas matemáticos. Por tanto, se presenta esta investigación documental con el objetivo de analizar una serie de factores asociados con la resolución de problemas de Matemática que permitan describir, comprender o explicar la razones por las cuales los estudiantes, y también sus docentes, tienen éxito, o fracasan, en el aprendizaje de la Matemática.

Para tal efecto, se utilizó la técnica de análisis de contenido, desde su arista cualitativa, a fin de analizar e interpretar la información textual contenida en diversos documentos, previamente publicados por medios impresos y electrónicos, que versan sobre la resolución de problemas matemáticos, orientando la mirada hacia lo afectivo y sin descuidar dominios como el cognitivo, metacognitivo, contextual o social, pues, en el momento de resolución entran en escena factores de distinta naturaleza. Desde allí, tendrá sentido analizar aspectos que tienen que ver con el éxito, o con el fracaso, de los estudiantes y de los docentes en cuanto al aprendizaje de contenidos matemáticos, lo cual abre espacio para abordar situaciones como las incompetencias matemáticas aprendidas, la ansiedad hacia la Matemática y la ausencia de resiliencia matemática, siendo esta última vital para arrostrar todo ese compendio de adversidades que han venido acumulándose en torno al proceso de enseñanza-aprendizaje-evaluación de los contenidos matemáticos.

\section{2. ¿Qué es un problema matemático?}

Cuando se está ante un verdadero problema matemático la situación que se plantea debe ser nueva y conflictiva, y su resolución no debe corresponderse con la aplicación mecánica de algoritmos preestablecidos que suelen aprenderse al momento de darse ejemplos o resolverse ejercicios que no trascienden el mero seguimiento de pasos ya dados con anterioridad. $\mathrm{Al}$ no tenerse un modelo de resolución inmediato ni disponerse de algoritmos que relacionen "los datos con las incógnitas o de un proceso que identifique automáticamente los datos con la conclusión" (Vila \& Callejo, 2004, p. 32), se está ante un verdadero problema.

Retomando lo anterior, en la Figura 1 se muestra una secuencia que suele guiar la presentación y aplicación de los constructos: ejemplo, ejercicio y problema, la cual es usual en muchas de las tradicionales clases de Matemática. Para sus acepciones, se siguieron las ideas señaladas por Martínez-Padrón y González (2007), y las correspondientes definiciones dadas por la Real Academia Española (2020). Por tanto, el encadenamiento se inicia considerando un determinado contenido matemático seguido de un modelo que ilustra un aserto que se propone para que se imite o se compruebe. Tal modelo se le conoce como ejemplo. Si luego de este, se plantean enunciados parecidos o semejantes, cuya resolución se corresponde con una prueba que se realiza, reiteradamente, mediante el manejo y la reproducción sistemática de algoritmos, métodos y reglas ya empleadas en el ejemplo dado, se está ante un ejercicio donde siempre es posible acometerlo siguiendo pasos previstos y aprendidos de otros ejercicios semejantes o parecidos a los desarrollados a continuación del ejemplo. 


\section{Figura 1. Secuencia usual de la presentación de los términos: contenidos, ejemplos, ejercicios y problemas}

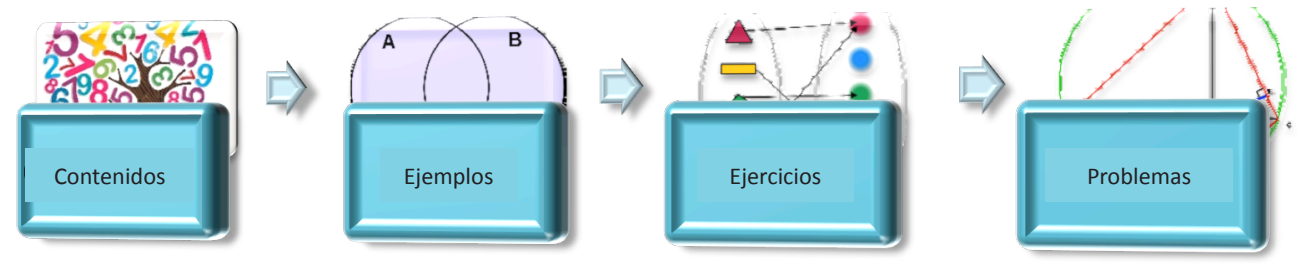

Nota: Figura construida con información tomada de Martínez-Padrón, O. J., \& González, F. (2007). Problemática de la formulación de problemas de Matemática: Un caso con docentes que enseñan Matemática en la Educación Básica venezolana, GEPEM, 50, 43-62.

Finalmente, aparece lo que se denomina un problema matemático que, por su naturaleza, obliga a trascender el uso de algoritmos ya conocidos por el resolutor y supone una situación nueva cuya respuesta es desconocida por este, pero puede obtenerla a través de métodos que demandan de la aplicación, no mecánica, de conocimientos matemáticos previamente establecidos, solicitados o construidos. Tal situación requiere de la heurística (Polya, 1965) representada por una forma de descubrimiento orientada por procedimientos inductivos y experimentales.

Lamentablemente, en las clases de Matemática suele acudirse a los ejercicios ya que los propios estudiantes, sus padres, la escuela y la sociedad acostumbran a solicitarle a los docentes, que mientras no le den algunos modelos de "problemas" a seguir, los estudiantes no podrán resolverlos, no dándose cuenta de que este proceder está en detrimento del aprendizaje de la Matemática (Martínez-Padrón, 2016). Tales agentes prefieren que los estudiantes aprueben, a que den muestras de las competencias matemáticas que realmente se requieren para resolver cualquier problema matemático. Por eso, es común encontrar que la clase tradicional de Matemática siempre esté llena de ejercicios, como los mencionados por Blanco Nieto y Pino (2015), que solo sirven para practicar rutinas, recordar propiedades, resolver ecuaciones o reconocer algoritmos y aplicarlos en ejercicios como los dados en los modelos.

Aunque todo problema debe cobijar la búsqueda de distintas estrategias de solución y obligar al uso de habilidades, conocimientos y estrategias más elaboradas, la denominación de problema o de ejercicio es relativa: depende del contexto, del momento o de si resulta novedoso o no para un eventual resolutor. Sobre esta última relatividad conceptual, Charnay (1993) expone que lo que puede ser un problema para un resolutor, puede no serlo para otros, pues, el conflicto o dificultad característica de cada problema no siempre es percibida por todos, o por algunos, de la misma manera; aunque hay que tener en cuenta que en aula acontecen situaciones que, de no hacerlas conscientes, pueden ser consideradas como inéditas, en algunos casos, y asumirlas como novedosas.

Charnay (1993) declara que todo problema debe "ofrecer una resistencia suficiente para llevar al alumno a hacer evolucionar los conocimientos anteriores, a cuestionarlos, a elaborar nuevos" (p. 61) y aunque su resolución debe corresponderse con un desafío intelectual, se hace necesario cuidarse de la construcción de su enunciado, puesto que el mismo tiene que ser comprensible y solucionable mediante la utilización del repertorio personal que se activa cuando se inicia el proceso de resolución.

Muchos son los factores que se activan, o se inhiben, antes, durante o después de que un estudiante se somete al proceso de resolución de cualquier problema, destacando aquellos de carácter cognitivo, metacognitivo, comportamental, social y afectivo. González (2020) sostiene que cuando el resolutor entra en escena involucra, al menos, el repertorio de conocimientos que tiene sobre la Matemática, 


\section{MARTÍNEZ-PADRÓN}

El afecto en la resolución de problemas de Matemática

sus pensamientos y lo afectivo, generando desde allí cuatro dimensiones: disciplinaria, cognitiva, metacognitiva y emocional. No obstante, parece necesario acoplar la denominación de la última dimensión como afectiva, en vez de emocional, asumiendo que lo afectivo es más abarcador y las emociones forman parte de él. Pero ¿Qué significa resolver un problema matemático?

\subsection{La resolución de problemas de Matemática}

En el ámbito popular, resolver un problema de $\mathrm{Ma}$ temática implica el encuentro de una solución única a una situación planteada, siguiendo los pasos dados por el profesor, pero esa creencia puede ser cuestionable.

Antes de hacer nuevas consideraciones, se hace necesario indicar que, independientemente del país, del sistema educativo, de la modalidad o las carreras de formación académica, los programas de Matemática y de otras áreas científicas, siempre solicitan resolver problemas, muchos apoyados con el lenguaje matemático.

La National Council of Teachers of Mathematics (2000) establece, en sus Principios y Estándares para la Educación Matemática, que "la resolución de problemas constituye una parte integral de todo el aprendizaje de las matemáticas" (p. 55), aseverando que la misma debería formar parte de los programas de enseñanza de todas los niveles y modalidades que configuran los sistemas educativos. También establece que la resolución de problemas no es solo un objetivo sino un medio para aprender Matemática, siendo una parte integral y no una pieza aislada. Por eso, recomienda usarla para que los estudiantes tengan muchas oportunidades para formular, lidiar y resolver problemas complejos que implican esfuerzos significativos. Igual permite: (a) reflexionar sobre el pensamiento que siguen los estudiantes durante la resolución; (b) adaptar y adoptar estrategias propicias para resolver otros problemas; (c) precisar formas de pensar y hábitos de persistencia; y (d) dar espacio a la curiosidad y a la confianza para abordar situaciones desconocidas que no necesariamente ocurren en el aula de Matemática.
Puede observarse que la inclusión de la resolución de problemas en la clase la Matemática es transcendental, dado que invita a poner a prueba todo el repertorio de potencialidades cognitivas, metacognitivas, comportamentales, sociales y afectivas que poseen los estudiantes, así como somete a prueba el conocimiento profesional de quien le enseña, puesto que con ello da cuenta de su capacidad didáctica, de sus competencias matemáticas y de todo aquello que garantice y permita la adquisición de conocimientos y la producción de saberes por parte de los estudiantes, aunque, se sigue teniendo dudas sobre la capacidad de muchos de docentes en estos quehaceres.

En vista de que siempre es necesario apoyar a los estudiantes en el proceso de construcción de conocimientos y saberes que les permitan resolver problemas matemáticos, no se puede negar la oportunidad de "experimentar la emoción de resolver un problema concreto, o de descubrir algo nuevo, o de encontrar por sí mismos un patrón que otros no advirtieron" (Síntesis Educativa, 2011), basta valerse de la utilidad y belleza propia de la Matemática, al momento de ser aplicada para resolver problemas. Experimentar esa emoción siempre ayuda a que los estudiantes tomen decisiones fundamentadas ya que les da la oportunidad de poner a prueba todas sus pericias resolutorias.

Estando establecidas algunas consideraciones sobre los problemas, es el momento de analizar algunos factores involucrados en el proceso de su resolución, acotando el análisis solo a los relacionados con el dominio afectivo.

\section{Factores Básicos del Dominio Afectivo}

Seguidamente, se establece lo que se entenderá por creencias, actitudes y emociones, asumiendo que tales factores representan los componentes básicos del dominio afectivo. No obstante, se agrega lo motivacional, en virtud de que Spinoza, citado por Damasio (2009), considera al afecto como un compendio de "impulsos, motivaciones, emociones y sentimientos” (p. 7). 


\subsection{Las creencias en la resolución de problemas matemáticos}

Las creencias siguen teniendo un impacto decisivo en el aprendizaje de la Matemática. Intentar resolver un problema matemático, o negarse a hacerlo, puede causar heridas psicológicas debidas a traumas matemáticos. También, el creerse torpe para resolver el problema, o ser víctima de lo que Flores (2013) denomina anomia asiliente, hace que broten perturbaciones o embotamientos que impiden hasta leer y comprender el enunciado de cualquier problema dado, pudiendo propiciarse la comisión de errores o generar un estado emocional como la ansiedad matemática.

En muchas situaciones escolares y sociales, se sostiene que la Matemática es difícil de aprender y esa creencia lacera el espíritu de los estudiantes y al de muchos de sus representantes quienes se conforman con que sus representados aprueben los cursos de Matemática, sin importarles que aprendan o no los contenidos correspondientes (Martínez-Padrón, 2016). Otras creencias concomitantes también los afligen, fundando la necesidad de generar soluciones a este y otros asuntos relacionados con dicha problemática, tanto desde los miembros de la noosfera que se han ocupado hasta de cambiar las programaciones a fin de que se mejore el aprendizaje de los contenidos matemáticos, como desde los investigadores de Educación Matemática, quienes han ido entendiendo que el continuo fracaso de los estudiantes, y también de sus docentes, de la escuela y del discurso utilizado en el aula y en la sociedad, continúa marcando importantes preocupaciones. Aunque todo eso trasciende el ámbito cognitivo, los cambios no han mejorado el aprendizaje de la Matemática ni lo que se piensa de ella..

Entre tantas interrogantes, hay quienes se preguntan ¿cómo pueden impactar los factores del dominio afectivo en la resolución de problemas matemáticos? Igual habrá quienes revisen muchos de esos hallazgos, encontrando que esos factores que impactan en el aprendizaje de la Matemática, no es solo un asunto de quien aprende, sino de quien enseña, evalúa o planifica. También depende cómo es vista por los estudiantes, por los docentes o por la sociedad, en general: ¿un conjunto de procedimientos sustentados en entes abstractos?, ¿una ciencia deductiva?, ¿patrones y pautas? ¿un lenguaje... el lenguaje de la ciencia?, ¿un referente cultural?

Estas y muchas otras nociones pueden emerger de lo que la gente piensa sobre lo que es la Matemática, pero, como ese no es el interés de este documento, no se abrirá espacio para esta discusión. Pero, cualquiera sea la acepción, una de las nociones que manejan los estudiantes, cuando les hablan de Matemática, es: números, formas, variables, propiedades, tablas, reglas, ejercicios, problemas, fórmulas y algoritmos (Martínez-Padrón, 2016), lo cual conforma el repertorio que suelen memorizar cuando tratan de resolver cualquier problema matemático. De ese repertorio, reproducen lo que recuerdan o involucran en su imaginación y en su creatividad para afrontar el problema que se les plantee. Pero, creer que pueden o no resolver dicho problema sigue siendo un asunto que mantiene preocupados a los protagonistas de la clase, en virtud de los compromisos que tales problemas acarrean.

Cuando un estudiante decide resolver cualquier problema matemático, se supone que lo primero que hace es leerlo y comprenderlo, para luego concebir un plan ejecutable que permita resolverlo hasta obtener la solución y, finalmente, examinarla (Polya, 1965). De plano, la primera etapa del problema solicita determinar incógnitas, datos y condiciones, así como contextualizarlo, pero si esto no ocurre, porque el estudiante cree que la Matemática es dificultosa, espinosa o complicada, quizás ni siquiera lea el enunciado, dejando para luego la responsabilidad de hacer la tarea.

Igual ocurre cuando el estudiante lee el problema y, por automatismo, cree que sigue el modelo de problemas anteriores y da rienda suelta a procederes que no siempre se corresponden con la naturaleza que enmarca este nuevo problema. Nótese que cuando se le atribuyen propiedades o califican a los problemas como difíciles, por ejemplo, pueden derivarse impulsos 


\section{MARTÍNEZ-PADRÓN}

El afecto en la resolución de problemas de Matemática

que confluyen de "diferentes fuentes que proporcionan elementos para la construcción, desarrollo, fortalecimiento, cambio, disminución o desaparición de creencias en relación con la escuela, con los docentes o con la Matemática y los procesos ligados a ella” (Martínez-Padrón, 2016, p. 29). Por ende, las creencias forman parte de ese sustrato que deben tener los estudiantes para resolver sus problemas en su quehacer escolar, pues, en la medida en que ellos proporcionan sus puntos de vista sobre el problema, se va elaborando ese fundamento conceptual que es de capital importancia, dado que impacta en sus pensamientos y en sus acciones (Martínez-Padrón, 2013, 2014).

Tal sustrato no sólo es personal sino social, al punto de que existen muchas otras creencias hacia la Matemática que siguen, aún, el mismo hilo planteado por Schoenfeld (1992) hace casi tres décadas y están asociadas con la experiencia escolar. A saber, su lista menciona creencias como las siguientes: (a) la Matemática se aprende siguiendo pasos que deben memorizarse y aplicarse de manera mecánica; (b) los problemas matemáticos tienen una solución única; y (c) existe una y solo una manera resolver los problemas matemáticos y la misma debe concordar siguiendo el modelo que el docente da en clase.

Tales creencias no cobijan la heurística planteada por Polya (1965), ni procesos de descubrimiento o de invención; tampoco fomentan la empatía ni la posibilidad de arriesgarse en una aventura para ver qué pasa si se pone en escena el compendio de conocimientos que se pueden tener en torno a la tarea de resolver un problema matemático.

Se asume, entonces, que las creencias constituyen un elemento del conocimiento que no siempre responden a la razón (Quintana, 2001), también provienen no solo de los sentimientos y deseos personales, sino de los grupos socioculturales, propios o ajenos, que las inducen socialmente. Por eso, se van gestando, consolidando o transformando a través de las experiencias de vida de cada sujeto (Callejo \& Vila, 2003) y "representan construcciones que el sujeto va elaborando en su proceso de formación para entender su mundo" (Martínez-Padrón, 2013, p. 231).
Se reconoce que las creencias se comportan como principios rectores y se asumen como los axiomas en la Matemática, pues, no requieren demostración alguna (Martínez-Padrón, 2008, 2013, 2014). Tales principios impactan en los contenidos que se movilizan en el aula y en todo lo que allí acontece, dando espacio, incluso, para consolidación, transformación o emanación de otras nuevas que también provienen de las experiencias que allí se producen. Todo esto es vital para cuando se gesten estrategias didácticas que utilicen como base la resolución de problemas matemáticos, dado que las creencias que los estudiantes tienen sobre Matemática provienen desde el mismo contexto social (Erazo \& Aldana, 2015).

Tomando en cuenta tales consideraciones, las creencias son "principios rectores que forman parte del conocimiento adquirido por los sujetos sobre la base de sus experiencias de vida. Tienen un carácter intersubjetivo y representan construcciones que, implícitamente, están presentes al momento de actuar ante el objeto o sujeto que las motivan" (Martínez-Padrón, 2016, p. 29).

\subsection{Las emociones en la resolución de problemas matemáticos}

García-González y Martínez-Padrón (2020) sostienen lo embarazoso que resulta establecer una definición universal sobre las emociones, así como separar sus elementos constituyentes, a sabiendas de su relación "con lo cultural, social, biológico, filosófico o psicológico” (p. 160). Si eso se observa desde la ansiedad, aversión o depresión que son muy comunes en la resolución de problemas, lo solicitado se vuelve más arduo de concretar.

Tampoco existen acuerdos que permitan observar una clara distinción entre los sentimientos y las emociones. Sin embargo, Damasio (2009) asume que "la emoción y las reacciones relacionadas están alineadas con el cuerpo, los sentimientos con la mente" (p. 7). Este mismo autor, citado por Pereira (2019), señala que:

las emociones incluyen determinados estilos de pensamiento y se corresponden con patrones específicos de activación fisiológica y comportamental, 
causados por el objeto de la emoción... [pero no] son una consecuencia de los sentimientos, sino que más bien son la causa de estos (p. 24).

Si se tiene presente que las emociones siempre están involucradas y comprometidas en la vida de cualquier sujeto, y que no es fácil encontrar alguna actividad donde no lo estén, hay que aceptar que comandan, prácticamente, todas las actividades que desempeñan los seres humanos. Es lógico deducir, también, que gobiernan las actuaciones de los estudiantes al momento en que resuelven cualquier problema matemático, habiendo casos que hasta secuestran (Goleman, 2006) la capacidad cognitiva. García-González y Martínez-Padrón (2020) indican que eso se debe a la presencia de emociones negativas que los impulsan a conductas contrarias a las esperadas y los alejan del problema que deben resolver, en vez de emprenderlo o afrontarlo, cultivando así una indefensión aprendida que consiste en no hacer nada ante el estímulo adverso.

Tal indefensión, Flores (2013) la asocia con la anomia asiliente que está consustanciada con la errada creencia de que no se es capaz de lograr algo, independientemente de que ese algo pudiera no tener alguna dificultad en sí. En Matemática, por ejemplo, ese algo pueden ser los problemas donde muchos estudiantes se inhiben y ni siquiera llegan a leer su enunciado porque, de antemano, ya dicen que no pueden con él y, en consecuencia, se desmotivan y no hacen esfuerzo alguno por afrontarlo. Muchos de estos casos podrían no tener ausencia de algunos conocimientos matemáticos para dar cara a la situación, pero su actitud, según Flores (2013), es enferma y sus conductas son desviadas de la norma, llegando a transformar la visión real de sí mismo en una incompetencia aprendida.

Existen premisas que atestiguan el rol preponderante de las emociones en la facilitación o inhibición del aprendizaje, teniendo mayor contribución que lo cognitivo (Goleman, 1996, 2006). En este conflicto, Solomon, citado en García-González y Martínez-Padrón (2020), advierte sobre la dificultad de dominarlas y sobre el riesgo en que ponen a sus implicados, pudiendo ser "más peligrosas y comprometidas que la propia razón de los sujetos, aunque esta última es quien debe controlarlas" (p. 159).

Cuando se habla de emociones, Vernaza-Pinzón et al. (2019) dan cuenta de que impactan los procesos cognitivos de los sujetos, afectando así su comprensión y su atención. Las catalogan como experiencias multidimensionales que incluyen "tres sistemas de respuesta: cognitivo/subjetivo, conductual/expresivo y fisiológico/adaptativo” (p. 149), aunque Cossini et al. (2017) las precisan en cinco componentes: expresión motora, cognitivo, neurofisiológico, motivacional y experiencia subjetiva, pero esto no será un asunto por precisar en este documento, dado que se advierte una buena intersección categorial.

Cuando suelen detectarse, por ejemplo, cambios fisiológicos, se precisan expresiones faciales particulares, alteraciones en la frecuencia cardíaca o presencia de sudoración corporal (Goleman, 1996; 2006) que se activan cuando el organismo detecta alguna situación inesperada que represente un riesgo o una amenaza, y eso le pueda causar inseguridad o inestabilidad.

Aunque cada persona experimenta sus emociones de manera particular, las reacciones son automáticas (Damasio, 2009; Cossini et al., 2017) y de difícil control, tal como ocurre en situaciones de miedo, irritación, aversión, angustia o tristeza que hacen que los aprendices de contenidos matemáticos abandonen, muchas veces, sus tareas e, incluso, el aula de clase de Matemática (Martínez-Padrón, 2016), pues, generan comportamientos, poco adaptativos, que se convierten en una amenaza que suele acompañarles hasta hacerlos acreedores de incompetencias matemáticas aprendidas que tanto daño causan a un importante contingente de aprendices que desertan de la clase de Matemática.

Resumiendo lo planteado, se puede concretar que las emociones tienen un carácter afectivo, de duración momentánea, siendo concebidas como un conjunto de

reacciones psico-fisiológicas que emite un sujeto en respuesta a un suceso, interno o externo, teniendo para él una carga de significado... suelen estar acompañadas de expresiones orgánicas 


\section{MARTÍNEZ-PADRÓN}

El afecto en la resolución de problemas de Matemática

características asociadas con pensamientos, motivaciones, experiencias, elementos hereditarios, cogniciones, estados psicológicos, estados biológicos y tendencias de actuar (Martínez-Padrón, 2016, p. 31).

Antes de perfilar otros factores del dominio afectivo, se hace necesario hacer referencia a una investigación realizada por García-González y Martínez-Padrón (2020) quienes presentan una reflexión derivada de una serie de experiencias que permitieron abordar el conocimiento emocional que posee un grupo de profesores de Matemática que participaron en un taller de Matemática Emocional (Perú) y en un curso de posgrado en Docencia de la Matemática (México).

La pesquisa, guiada por la Teoría de la Estructura Cognitiva de las Emociones, de Ortony, Clore \& Collins (García-González \& Martínez-Padrón, 2020), fue "fundamentada en la idea de que las emociones son desencadenadas por las valoraciones cognitivas que la gente hace de una situación de manera consciente o no" (p. 160). Se alineó bajo el paradigma fenomenológico-interpretativo, que permitió hurgar las vivencias narradas o dibujadas por los profesores ya señalados.

Entre los hallazgos destacan que ellos deben aprender a reconocer las emociones que experimentan sus estudiantes, y las situaciones que las desencadenan, a fin de asistirlos para que tengan una sana vida emocional que les permita arrostrar las adversidades que pudieran estar presentes en las clases de Matemática. Igual concluyen que el estudio de las emociones es una tarea que demanda un conocimiento emocional profundo que muchos profesores deben gestionar, máxime cuando se conoce la dificultad de dominar o controlar las emociones propias o ajenas (García-González \& Martínez-Padrón, 2020).

Este conocimiento es clave al momento de encontrarse con estudiantes marcados por una incompetencia aprendida que debe ser afrontada, con prontitud, habida cuenta de que el corazón de la Matemática son los problemas y resolverlos puede ser grato y divertido, sobre todo cuando se tiene afición por los mismos. Tal afición debe permitir moverse, con gusto y placer, entre interrogantes, sospechas, experiencias y sugerencias (Polya, 1965) que emergen desde el momento en que se comprende el enunciado del problema. Por tanto, quienes enseñan Matemática deben poseer un conocimiento emocional capaz de afrontar situaciones de estudiantes que no se atreven ni intentan siquiera leer y comprender lo que se solicita ser resuelto en el problema. También, deben atender aquellos que se atascan en el camino a fin de estimularlos a continuar luego, eso bajo la idea de que el encuentro de la solución del problema debe ser abordado con vehemencia, sin que ello esté motivado por recompensas exteriores, más que el logro de la respuesta correspondiente.

Bajo esa premisa, hay que dar a entender que ¡ ${ } \mathrm{No}$ siempre basta un solo día para aprender tocar, perfectamente, un instrumento! (Du Sauto, 2015), por lo que se debe dejar por sentado que el aprendizaje de algunas entidades matemáticas puede demorar, no solo por cuestiones cognitivas, sino por el fuerte componente emocional que debe ser abordado con prontitud.

Esa Educación Matemática debe tener presente el uso de tiempos adecuados para lidiar con los aspectos señalados, sobre todo con los afectivos que demandan la posesión de un conocimiento emocional, por parte del docente que enseńa Matemática, siendo útil el hecho de estar pendientes de las valoraciones cognitivas que suelen desencadenar las emociones que los aprendices exhiben cuando resuelven cualquier problema matemático, valiéndose para ello, por ejemplo, de la Teoría de la Estructura Cognitiva de las Emociones ya mencionada por García-González y Martínez-Padrón (2020) que demanda una buena carga de compromiso, perseverancia y determinación para construir un sendero que encauzaría, de manera consciente, a la mente de los estudiantes y los colocaría sobre puertos seguros y distantes de la confusión y el desorden.

Alineados con la necesidad de resolver el problema del aprendizaje de la Matemática y, por ende, el de la resolución de problemas matemáticos, investigadores como Sepúlveda et al. (2019) señalan que existen 
investigaciones internacionales que hacen mención al bajo rendimiento que se produce en el área de $\mathrm{Ma}-$ temática, lo cual puede atribuirse a causas como la falta de motivación y la frustración.

De seguro, que en las mejoras deseadas se han probado cambios de estrategias, modelos u otras opciones que impactan la transposición didáctica, pero, lamentablemente, no han surtido el efecto deseado. No obstante, se sigue necesitando mejorar todo lo que acontece en el aula de Matemática y, en este momento histórico, se apuesta a incorporar nuevos programas para el cambio, como el denominado Aprendizaje Social, Emocional y Ético (Aprendizaje SEE: SEE Learning) que emergió de la comunión de ideas del Dalai Lama y de un equipo de investigadores asociados con la Emory University, Atlanta, Estados Unidos, quienes están interesados en promover el aprendizaje SEE, en todos los contextos, así como en los diferentes niveles y modalidades de los sistemas educativos del mundo, incluyendo desde kindergarten hasta la educación superior.

La meta del Aprendizaje SEE es potenciar el florecimiento académico de todas las generaciones a atender, a través de "una regulación emocional positiva, el desarrollo de la compasión, y las habilidades interpersonales" (Emory University, 2019, p. 7). Por tanto, prevé la alfabetización ética (Richard, 2016) y emocional (Goleman, 1996, 2006; Goleman \& Senge, 2016) de los participantes, cultivando tanto habilidades cognitivas como las denominadas blandas (Heckman, 2012), a la luz de la teoría de la inteligencia emocional y la teoría de aprendizaje social (Goleman, 1996, 2006; Goleman \& Senge, 2016), acotando que esta última sostiene que los estudiantes "aprenden mucho a partir de la observación de los comportamientos que ven modelados" (Emory University, 2019, p. 127) por los otros sujetos de su entorno.

Lo que se refiere a las habilidades blandas debe ser tratado como cuestiones transversales y, preferiblemente, desde edades tempranas (Ortega, 2017), no pudiéndose descuidar lo que ocurre tanto en la educación formal como en la informal, pues, el desarrollo de esas habilidades socioafectivas imanta el comportamiento de los aprendices y los rasgos de su personalidad. En esa educación informal, no intencionada, el aprendizaje es influenciado sin necesidad de que existan condiciones especiales, pues, todo sucede de manera natural. Por tanto, no deben descuidarse los desencadenamientos emocionales que acontecen en relación con la resolución de problemas matemáticos, dado que ocurren, transcurren y discurren situaciones cognitivas y socioafectivas de vital interés.

Se establece que ese Aprendizaje SEE puede ser útil en cualquier contexto donde se pretenda "enseñar competencias sociales, emocionales y éticas, sin alejarse demasiado de cómo hoy se les enseńa a los estudiantes matemáticas" (Emory University, 2019, p. 10) u otras asignaturas del campo académico. Por tanto, el desarrollo de las actividades matemáticas debe hacerse con entusiasmo, determinación, pasión, respeto y curiosidad, mostrando, siempre, habilidades para moldear resilientes matemáticos durante todos los momentos de clase.

\subsection{Las actitudes en la resolución de problemas matemáticos}

La actitud representa uno de los factores del dominio afectivo que más se ha investigado desde hace varias décadas, aunque la mayoría ha sido abordada desde el paradigma positivista y guiadas por asociaciones o modelos predictivos donde se ponen en juego otras variables tales como rendimiento en Matemática y la ansiedad matemática, que han permitido encontrar relaciones importantes y pronosticar comportamientos desde una de esas variables con respecto a las otras. No obstante, el tema de las actitudes hacia la Matemática aún tiene mucho que ofrecer, sobre todo si las investigaciones se platean desde diferentes miradas epistemológicas.

No será el momento para tomar decisiones sobre cuáles son los componentes esenciales que se deben considerarse para estructurar a las actitudes, sobre todo cuando su dimensionalidad ha estado expuesta a variabilidad. Sin embargo, autores como Ubillos, Mayordomo y Páez (2004) dan a conocer algunos modelos como los señalados en la Figura 2. 


\section{MARTÍNEZ-PADRÓN}

El afecto en la resolución de problemas de Matemática

\section{Figura 2. Visiones sobre las actitudes}

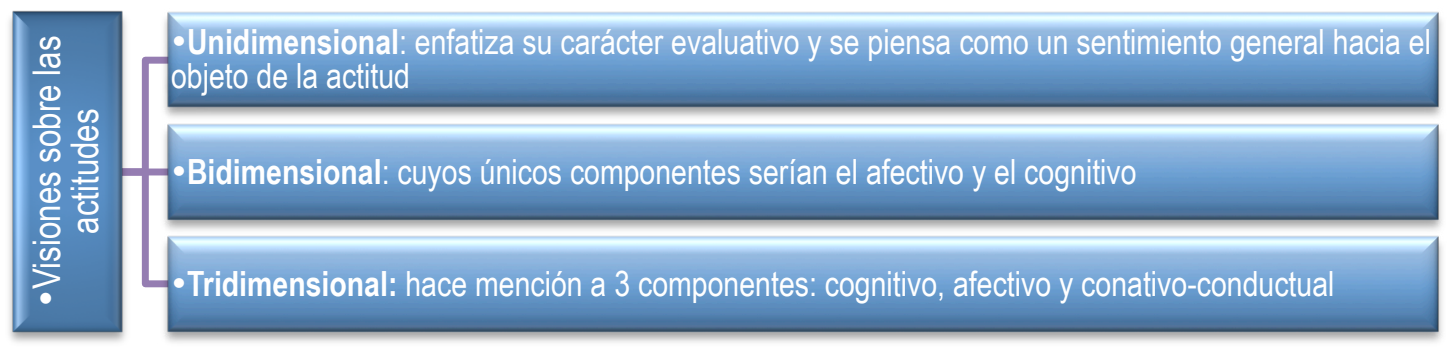

Nota: Figura construida con información tomada de Ubillos, S., Mayordomo, S., \& Páez, D. (2004). Actitudes: Definición y medición. Componentes de la actitud. Modelo de acción razonada y acción planificada. En D. Páez, I. Fernández, S. Ubillos, \& E. Zubieta (Eds.). Psicología social, cultura y educación (pp. 301-326). Madrid: Pearson Prentice Hall.

Autores como Gallego (2000), fracturan la dimensión conativo-conductual, ya mencionada en el modelo tridimensional, y la convierte en dos componentes: el conativo o intencional y el conductual o comportamental, obteniendo un modelo de cuatro dimensiones.

Lo siguiente ilustra una situación que puede ocurrir en una clase de Matemática, al momento que un docente solicita, al estudiante, resolver un problema matemático. Tal situación será visionada desde las dimensiones señaladas por Gallego (2000): Supóngase que, después de esa solicitud, un estudiante opina lo siguiente: "siempre he creido que resolver un problema de Matemática es algo muy difícil”. Esta creencia representa el componente cognitivo de la actitud que podría sentar las bases para que dicho estudiante se ponga en contra de una conducta esperada por su docente y manifieste, en consecuencia, que no le place ni le gusta resolver problemas de Matemática. Este sentimiento individual de rechazo, en relación con lo esperado, forma parte del componente afectivo de la actitud $y$, como consecuencia, el estudiante puede tender a no asistir a clase porque no desea enfrentarse a esta situación. Tal determinación pone de manifiesto un componente intencional y se percibe cuando toma la decisión de no asistir, formando parte de su voluntad que, por cierto, es consciente y vinculada con ese deseo que, finalmente, motiva una acción intencional y, por ende, conforma al componente comportamental.

Eso de las dimensiones aún queda abierto a discusión ya que el mismo Gallego (2000) también menciona un componente axiológico, debido a que la aceptación o el rechazo que puede tener cualquier individuo, hacia un objeto o situación, suele estar sujeto a valoraciones y otros factores concomitantes. Tal componente acostumbra a estar precedido de:

una valoración personal, no sólo si se está en condiciones de realizar el comportamiento perseguido, sino también en términos de los beneficios personales y comunitarios que los resultados de la actuación revierten...[Por tanto, tiene que ver con] los principios que permiten considerar si algo es o no es valioso para el sujeto y el razonar sobre cuáles son los fundamentos que sustentan el juicio de valor (Martínez-Padrón, 2008, p. 29).

Así como Gallego (2000) reestructura las dimensiones de las actitudes, Krech y Crutchfield (citados en Sarabia, 1992) también estiman lo de la motivación, partiendo de la acepción de que las actitudes son organizaciones duraderas "de procesos motivacionales, emocionales, perceptuales y cognitivos con respecto a algún aspecto del mundo del individuo" (p. 135). Siendo así, se puede concretar que, si lo motivacional no está presente en todo este complejo organizacional, no tendría sentido hablar de actitud, dado que este componente implica la presencia de lo afectivo y lo comportamental. Además, la actitud juega papeles preponderantes en el comportamiento motivado tanto de los estudiantes como de los profesores, siendo clave al momento de analizarse el proceso de enseñanza-aprendizaje-evaluación de los contenidos que configuran a los problemas matemáticos. 
Martínez-Padrón (2016) concreta que las actitudes son "reacciones valorativas o evaluativas manifiestas a través del agrado o desagrado hacia algún objeto, sujeto o situación” (p. 32), y este constructo sintetiza ideas tomadas de autores como McLeod (1992) y Gómez Chacón (2000) cuando reportan que las mismas:

- Predisponen al sujeto a la emisión de respuestas valorativamente preferenciales, orientando su pensamiento y adecuándolo al contexto.

- Dependen de creencias o emociones focalizadas en un objeto o situación.

- Se consideran como estados de ánimo que trascienden la mera cognición.

\subsection{La motivación en la resolución de problemas matemáticos}

Es común encontrar situaciones de aula donde los docentes se preguntan ¿Qué tendrían que hacer para que sus estudiantes logren motivarse y decidan resolver los problemas de Matemática que le son sugeridos?, ¿Cómo hacer para que resuelvan los problemas, con agrado, y se enamoren e interesen por la Matemática?

Desde 1945, George Polya sentía vital preocupación por eso de la resolución de problemas y publicó un libro titulado "How to solve It" que, en español, se tradujo en: "Cómo plantear y resolver problemas" (Polya, 1965), donde menciona varios factores del dominio afectivo, proponiendo que la solución de un problema de Matemática es una cuestión de voluntad y de determinación, trascendiendo lo meramente intelectual y poniendo en juego la esperanza y la satisfacción. Por lo que no es nuevo eso de ir más allá de la cognición, abriendo espacio para el rol que juegan las creencias, emociones, actitudes y motivaciones en todo ese proceso de resolución de problemas.

Como se observa, no se puede dejar de lado lo motivacional dada su contribución en el desarrollo de la vida académica de los estudiantes, pues, estar motivado impulsa acciones que persisten hasta lograr lo perseguido (Cespedes \& Martínez-Padrón, 2012).
Siendo así, la motivación se comporta como una palanca que direcciona la conducta, dando pie para definirla como un compendio de procesos implicados en la activación, dirección y persistencia de la acción, obligando a considerar tanto variables personales internas de los sujetos tales como intenciones, actitudes, percepciones, atribuciones, valoraciones, expectativas y representaciones que tenga sobre sí mismo y sobre la tarea que pretende realizar, como aquellas variables externas que tienen que ver con el contexto de acción en el que se desenvuelven (García \& Doménech, 1997).

Tal variedad de elementos, capaces de provocar, conducir, guiar y mantener el comportamiento de los estudiantes hacia el logro de determinados objetivos, está asociada con ese empuje o impulso que los conduce a resolver cualquier problema matemático, incluso con eficacia. Por tanto, el porqué de la ejecución de determinados comportamientos o por qué está en disposición de realizarlo suele estar explicado por medio de la motivación.

Conjugando autores como García y Doménech (1997), Flores (1999), y Cespedes y Martínez-Padrón (2012), se puede inferir que para resolver cualquier problema de Matemática es necesario que los resolutores no sólo puedan hacerlo, lo cual hace referencia a la posesión de ciertas capacidades, conocimientos, estrategias, pericias, habilidades y destrezas (componente cognitivo) para poder lograr la solución del problema, sino que también quieran hacerlo y, en este sentido, se toma en cuenta la disposición, la determinación, la audacia, la intención y la motivación (componente motivacional). Así, la resolución de cualquier problema no queda reducido solo al plano cognitivo, sino que obliga a considerar otros planos como los afectivos, sociales y contextuales, donde la motivación juega papeles estelares dentro de ese mundo de aficiones, intenciones, aventuras, metas y determinaciones. En todo caso, permite emprender, guiar, orientar y sostener determinados comportamientos necesarios para alcanzar las soluciones requeridas.

No cabe duda de que la motivación es una manifestación psicológica que puede inducir a los estudiantes a 


\section{MARTÍNEZ-PADRÓN}

El afecto en la resolución de problemas de Matemática

adquirir, desarrollar o sostener disposiciones favorables hacia el aprendizaje de contenidos matemáticos. Por ello, debe tomarse en cuenta cuando se aborda, por ejemplo, la falta de apetito para emprender tareas debido a la presencia de incompetencias matemáticas aprendidas. En este sentido, se recomienda apostar a la conformación de un compendio de factores que permitan formar, adiestrar o capacitar a los estudiantes para que puedan arrostrar, con determinación, las adversidades que suelen encontrar en los problemas que deben resolver hasta lograr su solución de manera exitosa. Eso conduce a la conformación de resilientes matemáticos, usando para ello las propias fortalezas cognitivo-afectivas-contextuales que brotan de la propia experiencia.

Según Miller-Karas (2015), la resiliencia se debe cultivar "a nivel cultural, estructural, interpersonal e individual” (p. 13), sustentando tal acción en el aprendizaje sistémico que también menciona Goleman \& Senge (2016), de manera que se pueda dar cara a cualquier desafío propio del mundo complejo y globalizado donde han de insertase los sujetos (Emory University, 2019).

Cuando en dicha resiliencia se afrontan situaciones adversas relacionadas con la Matemática, el constructo se denomina resiliencia matemática, el cual fue desarrollado por Johnston-Wilder y Lee (Johnston-Wilder, Lee, Brindley \& Garton, 2015) a fin de dar cuenta de la postura positiva que deben tener los estudiantes para aprender Matemática. Cuando se logra tal resiliencia, los estudiantes llegan a empoderarse de suficientes pericias para hacer frente a estados emocionales negativos, como la evitación, desgano, aversión, ansiedad e impotencia (Lee \& Johnston-Wilder, 2017), que suelen acompañarlos al momento de resolver cualquier problema matemático. Ellos sugieren que cuando los estudiantes presentan dificultades en Matemática hay que crearles una cultura de que sí son capaces de resolverlo, por lo que hay que afrontar esos obstáculos y derribar todas esas barreras que protagonizan el fracaso en Matemática.

Esta resiliencia matemática debe asumirse con el fin de superar los bloqueos cognitivo-emocionales que pueden emerger durante el proceso de enseñanza-aprendizaje-evaluación de los contenidos matemáticos, así como para clarificar posibilidades, fortalezas, experiencias y potencialidades hasta construir la correspondiente identidad del resolutor de problemas matemáticos. Por tanto, debería ser una práctica ubicua en todos los momentos y contextos donde se tiene la pretensión de decir que en esos espacios se enseńa y se aprende Matemática. Siendo así, se aspira configurar "ecologías del aprendizaje, entendidas como la base para los modelos educativos futuros de acuerdo con el contexto y las características del conocimiento actual: caótico, interdisciplinario y emergente (Martínez-Rodríguez \& Benítez-Corona, 2020, p. 44).

Entre las situaciones a confrontar, en el aula de Matemática, está la aversión que, según García-González y Martínez-Padrón (2020), está asociada con la ausencia de motivación, actitudes de rechazo y la repugnancia, lo cual trastoca, según García-González y Martínez-Sierra (2020), hasta la actuación de los docentes que enseñan la materia.

Para dar cara a las adversidades también es posible echar mano a la gamificación, vista como una técnica que permite organizar experiencias de aprendizaje capaces de motivar y de enseñar a los estudiantes en un espacio lúdico. Para atender problemas actitudinales, basta tomar en cuenta experiencias lúdicas como las propuestas por Cespedes y Martínez-Padrón (2012) en su libro sobre MatemáGica, el cual contiene actividades matemáticas diseñadas para despertar el interés y la motivación de los aprendices, al momento de participar en esas experiencias de aprendizaje que deben ser presentadas de manera asombrosa y mágica.

Sobre los juegos, el matemático Bill Barton también reportó, en una entrevista publicada por Síntesis Educativa (2011), que le gustaría ver más juegos matemáticos en las escuelas, admitiendo que las abstracciones del álgebra y la geometría pueden ser abordadas desde los juegos reglados. Asimismo, Cespedes y Martínez-Padrón (2012) abren espacio para las explicaciones algebraicas y para las demostraciones al exhibir, en su texto de MatemáGica, situaciones lúdicas enclavadas en curiosidades matemáticas de 
cualidad común. Tales situaciones fueron diseñadas para engendrar y disparar compulsiones interiores en los resolutores, acompañadas por la satisfacción de resolver, con vehemencia, cualquier problema, sin que esté motivado por recompensa externa alguna, más que la satisfacción personal de salir airoso por el logro de las metas (Colé y Colé, citado por Spinak, 1996; Cespedes \& Martínez-Padrón, 2012).

\section{Conclusiones}

A lo largo del discurso se da cuenta de la importancia que tienen los aspectos afectivos en el proceso de resolución de problemas matemáticos. Ese espectro es relevante, en virtud de que el éxito o el fracaso de los aprendices no siempre puede describirse, comprenderse o explicarse desde sus capacidades cognitivas, sino desde otros factores preponderantes del dominio afectivo. Por tanto, para resolver un problema matemático no basta con dominar los contenidos de la asignatura, también hay estar motivado y poseer creencias, emociones y actitudes favorables hacia lo que se hace y cómo se hace.

La carga cognitiva, metacognitiva, comportamental y motivacional también es válida de considerar en quienes tienen la responsabilidad de conducir el proceso de resolución de problemas matemáticos dado que, si estos no tienen el conocimiento profesional adecuado para conducir, con éxito, la tarea, también están destinados al fracaso, pues, corren el riesgo de no poder enseñar lo que no saben, o de no poder enseñar a amar lo que no aman.

Todas esas premisas obligan a que toda actividad que se perfile desde al aula de Matemática debe apostar al moldeamiento de resilientes matemáticos, en todo momento y contexto, norteando la mirada hacia una alfabetización emocional de los resolutores de problemas matemáticos, tomando en cuenta el aprendizaje social y sistémico. Tal abordaje debe ser tratado desde los primeros grados de escolaridad, requiriéndose un fortalecimiento del conocimiento profesional de quienes enseńan Matemática, así como la postura de hacerlo de manera transversal, tomando en cuenta las competencias socioemocionales, culturales y éticas de los aprendices de los contenidos matemáticos.
Finalmente, queda abierta la posibilidad de gestar los cambios hacia el mejoramiento del aprendizaje de la Matemática y, por ende, eliminar, de una vez por todas, los problemas de aversión y de incompetencia aprendida que siguen rondando al aula de clases de Matemática. Por tanto, es urgente manejar todo ese repertorio de factores cognitivos, metacognitivos, afectivos, éticos, comportamentales y motivacionales, a fin de afrontar, con voluntad y determinación, los tradicionales escollos que suelen encontrarse durante la resolución de cualquier problema matemático. Tal repertorio viabiliza la conformación de posturas a favor de la conformación de un importante contingente de resilientes matemáticos capaces de afrontar los desafíos que se les presentan durante la resolución de cualquier problema matemático.

\section{Referencias}

Blanco, L., \& Pino, J. (2015) ¿Qué entendemos por problema de matemáticas? En L. Blanco, J. Cárdenas, \& A. Caballero (Eds.), La resolución de problemas de matemáticas en la formación inicial de profesores de primaria (pp. 81-92). España: Universidad de Extremadura. Servicio de Publicaciones.

Callejo, M., \& Vila, A. (2003). Origen y formación de las creencias sobre la resolución de problemas. Estudio de un grupo de alumnos que comienzan la educación secundaria, Boletín de la Asociación Matemática Venezolana, 10(2), 173-194.

https://r.issu.edu.do/l.php?l =27E3W

Cespedes, G., \& Martínez-Padrón, O. J. (2012). La MatemáGica va a la escuela. Curiosidades matemáticas con un enfoque didáctico. Caracas: Fondo Editorial Universidad Pedagógica Experimental Libertador.

Charnay, R. (1993). Aprender (por medio de) la resolución de problemas. En C. Parra e I. Saiz (Eds.), Didáctica de las Matemáticas. Aportes y reflexiones (pp. 51-63). Buenos Aires: Paidós SAICP.

Cossini, F., Rubinstein, W , \& Politis, D. (2017). ¿Cuántas son las emociones básicas? Estudio preliminar en una muestra de adultos mayores sanos. Anuario de Investigaciones, 24, 253-257. 


\section{MARTÍNEZ-PADRÓN}

El afecto en la resolución de problemas de Matemática

Damasio, A. (2009). En busca de Spinoza. Neurobiología de la emoción y los sentimientos. Barcelona: Editorial Crítica S. L.

Du Sauto, M. (2015). ¿De verdad todos podemos ser genios matemáticos? $B B C i$ Wonder.

http://r.issu.edu.do/l.php?l=29bIh

Emory University (2019). Aprendizaje social, emocional \& ético. Atlanta: Center for Contemplative Science and Compassion-Based Ethics Attention: SEE Learning, SEE Learning, Emory University. https://r.issu.edu.do/l.php?l=174Tol

Erazo, H. J., \& Aldana, B. E. (2015). Sistema de creencias sobre las matemáticas en los estudiantes de educación básica. Praxis, 11, 163-169.

Flores, C. (1999). Motivación, una alternativa para el éxito. Venezuela: Fondo Editorial, Universidad Pedagógica Experimental Libertador

Flores, D. (2013). La resiliencia nómica. Mejor desempeño para una vida más exigente. México: Instituto Internacional de Investigación para el Desarrollo.

Gallego, R. (2000). Los problemas de las competencias cognoscitivas. Una discusión necesaria. Colombia: Universidad Pedagógica Nacional.

García, F., \& Doménech, F. (1997). Motivación, aprendizaje y rendimiento escolar. Revista Electrónica de Motivación y Emoción 1.

https://r.issu.edu.do/l.php?l=30T9V

García-González, M., \& Martínez-Padrón, O. J. (2020). Conocimiento profesional de los profesores de Matemáticas. Revista Educación Matemática, 32(1), 157-177.

https://doi.org/10.24844/EM3201.07

García-González, M., \& Martínez-Sierra, G. (2020). The history of a teacher's relief of his mathematics anxiety: the case of Diego. Educational Studies in Mathematics, 103, (273-291).

https://doi.org/10.1007/s10649-020-09941-8

Goleman, D. (1996). La inteligencia emocional. Buenos Aires: Javier Vergara Editor.
Goleman, D. (2006). Inteligencia social. La nueva ciencia de las relaciones humanas. Barcelona: Kairós.

Goleman, D., \& Senge, P. (2016). Triple Focus: Un nuevo acercamiento a la educación. Barcelona, España: Grupo Zeta, Ediciones B.

Gómez Chacón, I. (2000). Matemática emocional. Los afectos en el aprendizaje matemático. España: Narcea, S.A., Ediciones.

González, F. E. (2020). “El corazón de la Matemática" en la formación de futuros profesores de Matemática. Revista de Educação Matemática, 17, 1-25. https://doi.org/10.37001/remat25269062v17id245

Halmos, P. (2018). The heart of Mathematics. The American Mathematical Monthly, 87(7), 519-524. https://doi.org/10.1080/00029890.1980.11995081

Heckman, J., \& Katuz, T. (2012). Hard evidence on soft kills. National Bureau of Economic Research.

Johnston-Wilder, S., Lee, C., Brindley, J., \& Garton, E. (2015). Developing mathematical resilience in school-students who have experienced repeated failure. En 8th annual International Conference of Education, Research, and Innovation. Seville, Spain.

Lee, C., \& Johnston-Wilder, S. (2017). The construct of Mathematical Resilience. En U. Xolocotzin (Ed.), Understanding Emotions in Mathematical Thinking and Learning. Elsevier (pp. 269-291). United Kingdom: Academic Press. https://doi.org /10.1016/B978-0-12-802218-4.00010-8

Martínez-Padrón, O. J. (2008). Actitudes hacia la Matemática. Sapiens, 9(2), 237-256.

Martínez-Padrón, O. J. (2013). Las creencias en la Educación Matemática. Revista Educere, 57, 231-239.

Martínez-Padrón, O. J. (2014). Sistema de creencias acerca de la Matemática. Revista Actualidades Investigativas en Educación, 14 (3), 1-28.

Martínez-Padrón, O. J. (2016). ¿Qué dicen los docentes paraguayos en cuanto al afecto en el aprendizaje de la Matemática?: Una mirada desde el Curso Nanduti. Revista UNIÓN, 5; 24-43. 
Martínez-Padrón, O. J., \& González, F. (2007). Problemática de la formulación de problemas de Matemática: Un caso con docentes que enseñan Matemática en la Educación Básica venezolana. GEPEM, 50, 43-62.

Martínez-Rodríguez, R., \& Benítez-Corona, L. (2020). La ecología del aprendizaje resiliente en ambientes ubicuos ante situaciones adversas. Revista Comunicar, 28(62), 43-52.

https://doi.org/10.3916/C62-2020-04

McLeod, D. (1992). Research on affect in mathematics education: A reconceptualization. En D. A. Grouws (Ed.), Handbook of research on mathematics teaching and learning (pp. 575-596). New York: MacMillan Publishing Company.

Miller-Karas, E. (2015). Building resilience to trauma: The trauma and community resiliency models. Routledge.

National Council of Teachers of Mathematics (2000). Principles and standards for school mathematics. USA: Library of Congress Cataloguing-in-Publication Data.

Ortega, C. (2017). Desarrollo de habilidades blandas desde edades tempranas. Guayaquil: Colección ECOTEC. http://r.issu.edu.do/l.php?l=1752IV

Pereira, S. (2019). Emociones, intencionalidad y racionalidad práctica. William James y Antonio Damasio. Ideas y Valores, 68(170), 13-36.

https://doi.org/10.15446/ ideasyvalores.v68n170.77686

Polya, G. (1965). Cómo plantear y resolver problemas. México: Editorial Trillas.

Quintana, J. (2001). Las creencias y la educación. Pedagogía cosmovisional. España: Empresa Editorial Herder, S. A.

Real Academia Española (2020). Diccionario de la lengua española. http://www.rae.es
Richard, L. (2016). Promoting secular ethics, En J. Helliwell, R. Layard, \& J. Sachs (Eds.), World Happiness Report, 1. New York: Sustainable Development Solutions Network.

Sarabia, B. (1992). El aprendizaje y la enseñanza de las actitudes. Madrid: Aula XXI. Grupo Santillana de Ediciones, S.A.

Schoenfeld, A. (1992). Learning to think mathematically: Problem solving, metacognition, and sense-making in mathematics, En D. Grows (Ed.), Handbook for research on mathematics teaching and learning (pp. 334-370). New York: MacMillan.

Sepúlveda, A., Díaz-Levicoy, D., \& Minte, A. (2019). Deficiente rendimiento en matemática: análisis desde la perspectiva de los alumnos de Educación Básica chilena. Revista Espacios, 40(23), 1-27.

Síntesis Educativa (3 de Mayo, 2011). Entrevista a Bill Barton: enseñando a amar las matemáticas. Periodismo Educativo. https://r.issu.edu.do/l.php?l=34J27

Spinak, E. (1996). Diccionario enciclopédico de Bibliometría, Cienciometría e Informetría. Caracas: UNESCO CII/II.

Ubillos, S., Mayordomo, S., \& Páez, D. (2004). Actitudes: Definición y medición. Componentes de la actitud. Modelo de acción razonada y acción planificada. En D. Páez, I. Fernández, S. Ubillos, y E. Zubieta (Eds.), Psicología social, cultura y educación (pp. 301-326). Madrid: Pearson Prentice Hall.

Vernaza-Pinzón, P., Posadas-Pérez, L., \& Acosta-Vernaza, C. (2019). Dolor y emoción, una reflexión para el profesional en ciencias de la salud. Duazary, 16(1), 145-155.

http://dx.doi.org/10.21676/2389783X.2639

Vila, A., \& Callejo, M. (2004). Matemáticas para aprender a pensar. El papel de las creencias en la resolución de problemas. España: Narcea, S. A. de Ediciones.

\section{CÓMO CITAR:}

Martínez-Padrón, O. J. (2021). El afecto en la resolución de problemas de Matemática. RECIE. Revista Caribeña de Investigación Educativa, 5(1), 86-100. https://doi. org/10.32541/recie.2021.v5i1.pp86-100 